\title{
Estructuras desplegables de aspas para cubiertas inclinadas
}

\section{Scissor-hinged deployable structures for inclined roofs}

\author{
M. J. Freire-Tellado ${ }^{(*)}$, M. Muñoz-Vidal ${ }^{(*)}$, I. López-César $^{(*)}$, J. Pérez-Valcárcel $^{(*)}$
}

\section{RESUMEN}

La atención a la evacuación del agua de lluvia y a la protección de la estructura ante los agentes meteorológicos ha dado lugar a un conjunto de propuestas de estructuras desplegables de aspas sobre planta rectangular en las que estas estructuras definen limas y faldones de pendiente suficiente. El trabajo parte de la resolución del trazado de estructuras desplegables de aspas planas simétricas y base cuadrangular con directriz quebrada, garantizándose el correcto funcionamiento cinemático mediante la construcción de modelos. Se estudian desplegables con directrices poligonales, curvas compuestas y también combinaciones de curvas y rectas. Se repasan los problemas de estabilización de la solución en el estado desplegado, incorporando la cobertura textil y cables de arriostramiento. Finalmente se desarrollan y analizan geométrica y cinemáticamente una serie de propuestas con superficies de intradós y extradós diferentes y otro grupo de ellas en las que los sentidos de despliegue no siguen los ejes del recinto a cubrir.

Palabras clave: Estructuras ligeras, estructuras desplegables, estructuras de aspas, secuencia de despliegue, diseño conceptual, morfología.

\section{ABSTRACT}

The attention to rainwater evacuation and to the structure protection against meteorological agents has given rise to a set of proposals of pantograph-based deployable structures on rectangular plant, in which these structures define limes and roof planes of sufficient slope. This work starts on the resolution of the layout of deployable structures with a broken guideline, guaranteeing the correct kinematic functioning through the construction of models. Polygonal guidelines, compound curves and also combinations of curves and straight lines are studied. The stabilization problems of the solution in the deployed state are analyzed, incorporating the textile cover and bracing cables. Finally, a set of proposals with different intrados and extrados surfaces and another group of them in which the deployment directions do not follow the two plan axis, are developed and analysed too.

Keywords: Lightweight structures, deployable structures, scissor-hinged mechanism, deployment sequence, conceptual design, morphology.

(*) Departamento de Construcción y Estructuras Arquitectónicas, Civiles y Aeronáuticas. E.T.S. Arquitectura, Universidad de A Coruña (España).

Persona de contacto/Corresponding author: manuel.freire.tellado@udc.es (M. J. Freire-Tellado).

ORCID: https://orcid.org/oooo-0oo2-8101-0088 (M. J. Freire-Tellado); https://orcid.org/oooo-0003-0479-1279

(M. Muñoz-Vidal); https://orcid.org/oooo-0002-4403-2368 (I. López-César); https://orcid.org/oooo-0002-6440-5432 (J. Pérez-Valcárcel)

Cómo citar este artículo/Citation: Freire-Tellado, M. J.; Muñoz-Vidal, M.; López-César, I.; Pérez-Valcárcel, J. (2019). Estructuras desplegables de aspas para cubiertas inclinadas. Informes de la Construcción, 71(556): e311. https://doi.org/10.3989/ic.64120.

Copyright: ( ) 2019 CSIC. Este es un artículo de acceso abierto distribuido bajo los términos de la licencia de uso y distribución Creative Commons Reconocimiento 4.0 Internacional (CC BY 4.0). 


\section{INTRODUCCIÓN}

Se entiende por estructuras desplegables de barras aquellos conjuntos de piezas articuladas que pueden transportarse en paquetes compactos que no ocupan más espacio que el del propio haz de barras concentrado y que, por procedimientos manuales o mecánicos, pueden desplegarse en los lugares en donde se requiera su uso para cerrar o crear recintos arquitectónicos, de instalaciones o constituir elementos auxiliares (1).

En sentido estricto se trata de mecanismos que no adquieren su rigidez hasta que, tras el despliegue, se restringe el exceso de grados de libertad que presentan, momento a partir del cual empiezan a funcionar propiamente como estructuras.

Algunas de ellas son de uso cotidiano: sillas de tijera, paraguas y sombrillas se encuadran en esta categoría y en ellos se encuentran hermosas soluciones de mecanismos estructurales; las plataformas elevadoras son una maquinaria de empleo usual...

Es común clasificar las estructuras desplegables (1) (2) en soluciones tipo paraguas (mecanismo deslizante), tipo mecanismo con articulaciones bloqueables -hinged-collapsible-strut mechanism, (2), p. 3-y soluciones con articulaciones pasantes formando aspas móviles, simples o compuestas. En el campo estructural estas últimas han recibido una atención continuada, y se han desarrollado soluciones para construir techos planos, cilíndricos, mallas esféricas, geodésicas o tipo Lamela (3) (4) y recientemente han sido propuestas soluciones elipsoidales (5).

Entre las primeras referencias de estructuras desplegables se encuentran las contenidas en los dos Códices de Madrid de Leonardo da Vinci -fechados entre 1491-1493 y 1503-1505(6) y la célebre escalera de Valturio $-1483-(7)$, Figura 1, que, si las fechas anteriores son correctas, sería la primera referencia histórica conocida.

En el desarrollo de las estructuras desplegables fue fundamental la figura del español Emilio Pérez Piñero (8), quien en los años 60 y 70 desarrolla estructuras desplegables planas y cúpulas pseudo-esféricas.

El sistema mereció la atención de la NASA, bajo cuyo auspicio se desarrollaron varias propuestas entre las que destaca el sistema de bastidor estructural para soporte de los módulos que conforman la estación espacial de 1990, desarrollado por el MIT en el otoño de 1985 -(2), pp. 15-16, 61-69-. Posteriormente You \& Pellegrino desarrollaron investigaciones de desplegables con aplicación aeroespacial: mástiles, estructuras desplegables anulares de aspas... que no pueden encuadrarse adecuadamente sin los trabajos de Hoberman, anteriores en el tiempo.

También son interesantes los trabajos de Ziegler a mediados de los setenta y Charis Gantes en el cambio de milenio, sobre estructuras desplegables autoestabilizadas.

En la actualidad existen propuestas de entidades como la Universidad de Nôtre Dame, Oxford Space Structures o incluso empresas como Ten Fold Structures. También se encuentran soluciones para pasarelas peatonales como el puente peatonal transformable Jet d'eau de Ginebra de Adrien Barakat.

En España se han llegado a construir algunos ejemplos, como la cubierta de la piscina de San Pablo de Sevilla de Escrig, Valcárcel y Sánchez (9), o las carpas desplegables de F. Blan-
DE RE MILITARI LIB. $X$

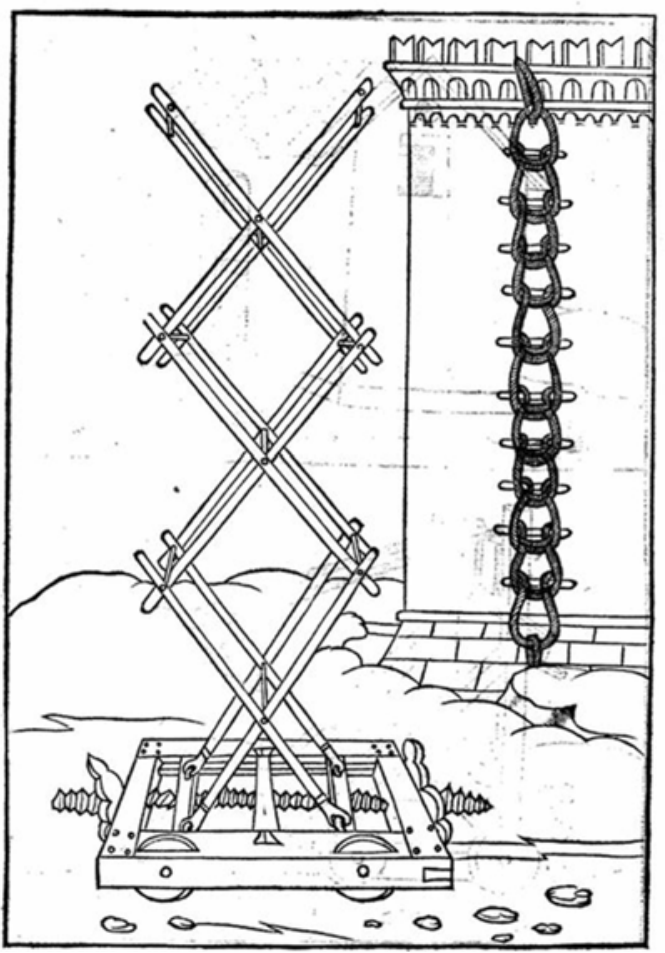

Figura 1. Escalera de Valturio.

co (10), y es cotidiano su empleo como soportes publicitarios transportables.

\section{LA EVACUACIÓN DEL AGUA DE LLUVIA: INFLUENCIA DE LOS AGENTES METEOROLÓGICOS}

El rápido montaje de las estructuras desplegables las hace muy interesantes para resolver situaciones sobrevenidas, como soluciones de emergencia -refugios, hospitales...-, lo que ha llevado a incluirlas en el proyecto de investigación 'Edificaciones de emergencia para situaciones de catástrofe humanitaria' (Referencia BIA2016-79459-R). Estas situaciones también se están produciendo en el primer mundo -recuérdese el caso de Calais-, y si bien todavía no se han identificado sus singularidades en estos casos, desde estas líneas se apuntan dos rasgos: una disponibilidad económica menos acuciante y una climatología en la que las precipitaciones no pueden ser olvidadas.

Las condiciones económicas de los países desarrollados permiten afrontar los costes derivados de una solución a base de estructuras desplegables, pero las condiciones meteorológicas imperantes han de ser tenidas en cuenta en el diseño: es preciso proteger tanto a los usuarios como a la propia estructura de los agentes climatológicos externos. En el caso de las desplegables de aspas, la construcción de una doble piel -tendiendo sendos textiles por la parte superior e inferior de la malla- crea una capa de aire intermedia que podría llegar a ser acondicionada, y ofrece una oportunidad para resolver el problema. Sin embargo, las condiciones de evacuación de las aguas pluviales imponen una condición de otro tipo: las desplegables se resuelven en la actualidad con directrices con pendiente nula en el punto de mayor cota, lo que podría -especialmente con coberturas deformables como las textilesdar lugar a situaciones de acumulación de agua que conduci- 
rían inexorablemente al hundimiento de la estructura puesto que se trata de estructuras muy ligeras.

Pérez Piñero proyectó la versión prismática de sus famosos teatros desmontables con cubierta plana a un agua (8). La solución geométrica para este tipo de desplegable es muy sencilla; en el caso de una propuesta de base cuadrada y aspas simétricas, el techo se formaría a partir de la repetición de la misma aspa en dos direcciones ortogonales del plano, con el único problema de fijar la estructura en la posición de despliegue deseada.

Sin embargo, desde el punto de vista de la evacuación de pluviales, son superiores las soluciones a varias aguas. En el caso más sencillo -dos aguas- la solución exige una directriz quebrada, esto es, la existencia de un vértice.

El profesor Sánchez Cuenca (11) propuso diversas configuraciones de estructuras desplegables de aspas con canto constante obtenidas por traslación vertical de la directriz, que permiten realizar quiebros en la cubierta. Sin embargo, las soluciones generadas por traslación se obtienen mediante sistemas de aspas asimétricas que, en la posición plegada, dan lugar a paquetes escalonados y, en general, no resuelven la formación de cerramientos verticales.

Para salvar estas limitaciones se planteó la introducción de un vértice en estructuras desplegables de aspas simétricas. Esta introducción incumple una de las condiciones -la continuidad de la directriz- establecidas por Escrig (3) para garantizar el despliegue de las mallas desplegables de aspas planas -se asume que cada sistema de aspas está contenido en un mismo plano-y que se resumen a continuación (Figura 2):

- Los nodos - puntos de corte de las aspas-deben estar contenidos en la curva directriz

- Los nudos -articulaciones entre pares de aspas- deben estar sobre una misma perpendicular a la directriz.

- El romboide formado al enlazar las aspas por sus extremos debe satisfacer la condición de que las sumas de las longitudes de los brazos de cada ' $x$ ' deben ser iguales, esto es , $l_{1}+l_{2}=l_{1}^{\prime}+l_{2}^{\prime}$

La investigación desarrollada demuestra que el incumplimiento indicado anteriormente es resoluble.

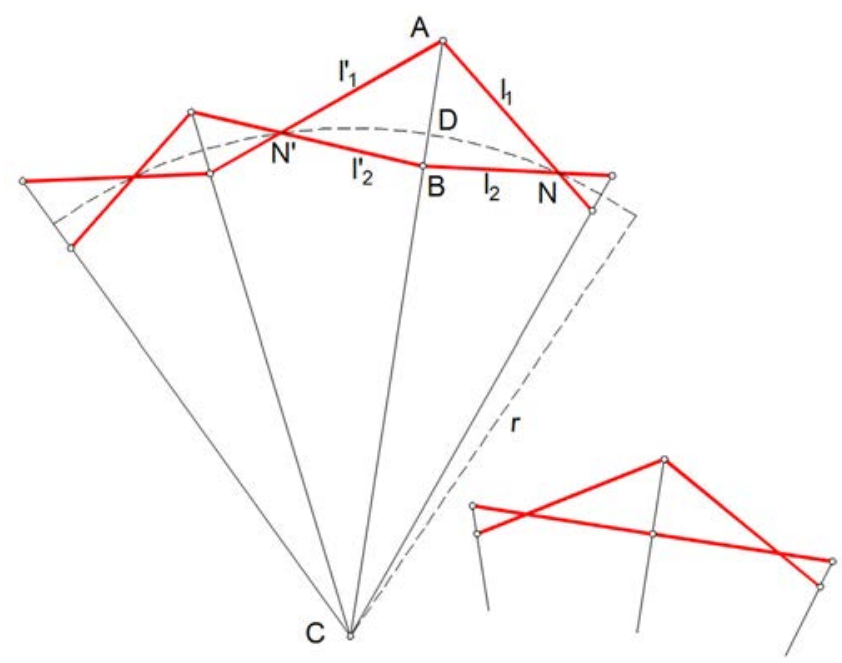

Figura 2. Condiciones de plegabilidad. Situación de acodalamiento.

\section{DESPLEGABLES DE DIRECTRIZ POLIGONAL}

En el caso de desplegables de base cuadrangular, aspas simétricas y ejes de giro horizontales, para realizar las pendientes se inclina uno de los conjuntos de aspas anteriores (el principal o aspas portantes), mientras que el perpendicular se mantiene horizontal (aspas transversales o de arriostramiento). El cambio de pendiente da lugar a un quiebro en la directriz para cuya solución es preciso el empleo de aspas asimétricas y de unas aspas de arriostramiento específicas.

Los trabajos desarrollados han establecido que para cada ángulo de encuentro y módulo de aspas empleados existe una -y sólo una- solución que da respuesta a las condiciones de igualdad de distancias longitudinales y transversales.

El estudio de la solución añade una limitación de diseño por acodalamiento de las barras -barras situadas en prolongación- incluso en faldones planos, derivada de la resolución del vértice, como se puede apreciar en la Figura 2, abajo.

La viabilidad del encuentro entre faldones planos se comprobó experimentalmente mediante el modelo de la Figura 3, que combina módulos estándar con módulos específicos para

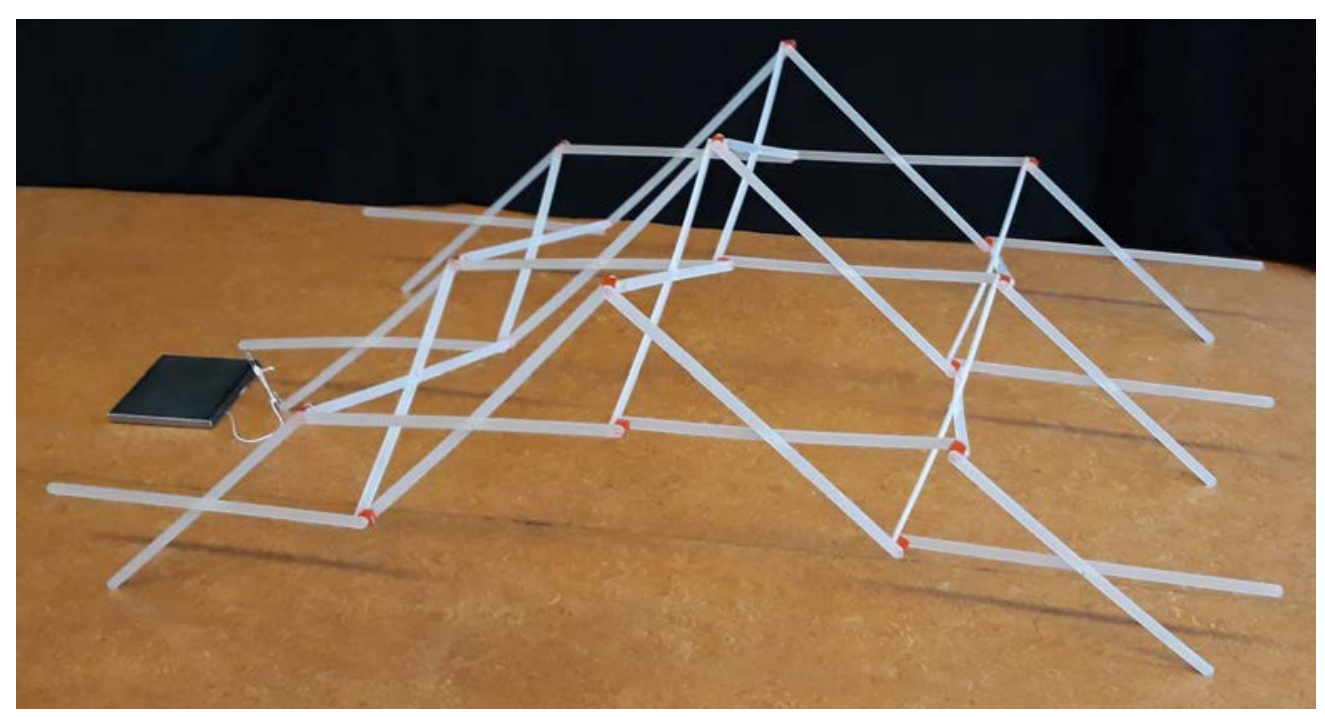

Figura 3. Solución del vértice en una estructura desplegable. 
resolver el quiebro. Está formado por tres planos de aspas principales enlazados por tres grupos de aspas transversales de arriostramiento. Cada uno de los planos de aspas principales está compuesto por sendas aspas estándar en los extremos y dos aspas asimétricas en el centro para resolver el cambio de pendiente; de forma similar, todas las aspas transversales son estándar salvo las del plano central.

Las barras del modelo se obtienen mediante corte láser de planchas de metacrilato de $4 \mathrm{~mm}$ de espesor y los nudos -cubos abiertos por una de sus caras- se conforman mediante impresión 3D. Las cuatro caras laterales reciben las barras de las aspas y la quinta se destina a la fijación de la cobertura textil.

La solución anterior permite la construcción de desplegables formadas a partir de haces simétricos con directrices poligonales, con perfiles triangulares, cuadrangulares..., si bien introduce una cierta apariencia conopial (concavidad hacia el exterior) en los módulos de acuerdo.

Se pueden emplear distintos números de módulos en cada lado de la directriz y distintos ángulos de encuentro entre tramos, si bien el plano del arriostramiento es necesariamente el plano bisectriz (Figura 4). Incluso en el caso de emplear un único módulo por faldón el planteamiento anterior permite resolver adecuadamente el encuentro con el suelo, como puede apreciarse en la Figura 4, derecha, dado que el arranque de la desplegable formando un cierto ángulo es el mismo problema anterior. Nótese no obstante que cada ángulo de encuentro diferente introduce dos nuevos módulos distintos - uno longitudinal y otro transversal- en la estructura.

En la Figura 4 se representan la directriz de cada trazado, las aspas principales y las aspas transversales -a trazos-, la verificación de las condiciones de plegabilidad y las superficies envolventes interiores y exteriores resultantes - mitad izquierda de cada figura-, y los distintos tipos de módulos necesarios para su construcción -mitad derecha-.

Los módulos asimétricos introducidos presentan diferencias de longitudes pequeñas, por lo que apenas afectan a la compacidad de la solución en situación plegada: las barras que concurren en el nudo interior quedan ligeramente despegadas del suelo y las del nudo superior, resaltadas del resto.

\section{DESPLEGABLES CILÍNDRICAS CON DIRECTRIZ FORMADA POR UNA ÚNICA CURVA}

Si se desplaza el nodo de un aspa simétrica de tal forma que ambas barras sean iguales pero queden divididas en dos partes distintas entre sí, se genera un módulo que repetido da lugar a una estructura curva. Si todos los módulos son iguales, el resultado es un arco de circunferencia, puesto que la curvatura resulta constante. Por ello la circunferencia es la directriz más recurrida, dado que la desplegable resultante se construye repitiendo una misma aspa, que además tiene ambas barras iguales, por lo que se precisa únicamente una barra para su construcción. El tipo de aspa empleado se denomina polar por cuanto los nudos de contacto entre aspas están situados sobre los radios de la circunferencia directriz (módulos M1 de la Figura 5).

En las soluciones cilíndricas con apertura inferior a $180^{\circ}$, los nudos extremos se sitúan a distinta altura, el apoyo se realiza sólo sobre uno de ellos y el aspa transversal queda en el aire (Figura 5, izquierda). La adicción de un módulo recto tangente y la aplicación de la solución anterior permiten conseguir que ambos nudos extremos se sitúen a igual cota y que el aspa transversal quede contenida en el mismo plano horizontal (Figura 5, derecha), resolviendo los problemas geométricos y las condiciones de plegabilidad transversal.

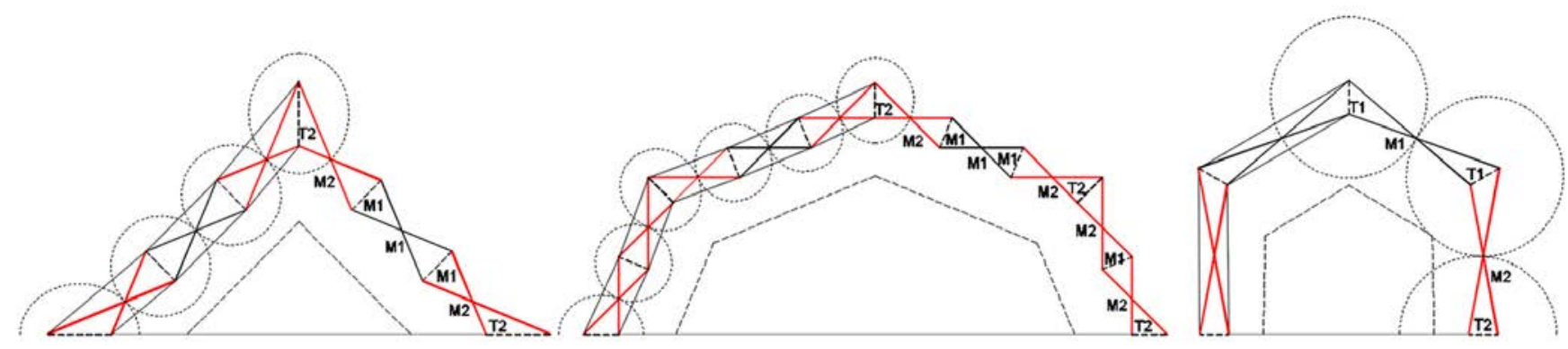

Figura 4. Estructuras desplegables de directriz poligonal.

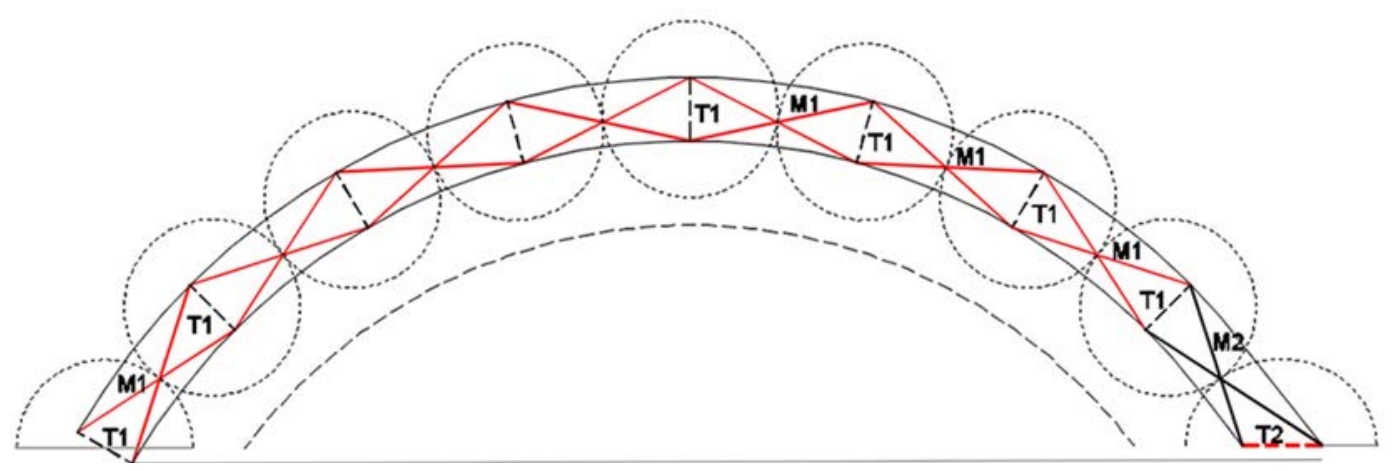

Figura 5. Comparación de arranques de desplegables de directriz rebajada. 


\section{DESPLEGABLES CILÍNDRICAS DE DIRECTRIZ FORMADA POR COMBINACIONES DE CURVAS (CURVAS COMPUESTAS)}

Para formar un recinto la cubierta ha de situarse a una cierta altura del suelo. Es evidente que esta distancia se puede resolver de varias formas, pero resulta atractivo que se integre dentro de la solución desplegable. Con directrices circulares y luces moderadas, para generar el recinto habitable la apertura tiene que ser próxima a $180^{\circ}$, pero en esas condiciones la solución cobra una altura excesiva. Para resolver este problema se ha recurrido a combinaciones de curvas tangentes en el punto de contacto -curvas combinadas o compuestas-.
Se han estudiado dos grupos de soluciones (Figura 6): el primero incluye las formadas por un medio óvalo -desplegable carpanel (Figura 6, arriba)-, alternativa geométrica a las elipses a lo largo de la historia de la construcción, y el segundo aquellas otras que combinan arranques circulares y uno o varios tramos rectos, introduciendo entre ellos uno o varios vértices -desplegables Tudor (Figura 6, abajo)-.

Existe un tercer grupo, el formado por arranques rectos con un tramo central curvo -arco de circunferencia-, que se obtiene rotando $90^{\circ}$ la solución anterior y completándola por simetría, pero que se puede generalizar a situaciones asimétricas (Figura 7). Si se eliminan los módulos rectos intermedios, la situación se convierte en la rebajada con arranques horizontales de la Figura 5 (derecha) anterior.

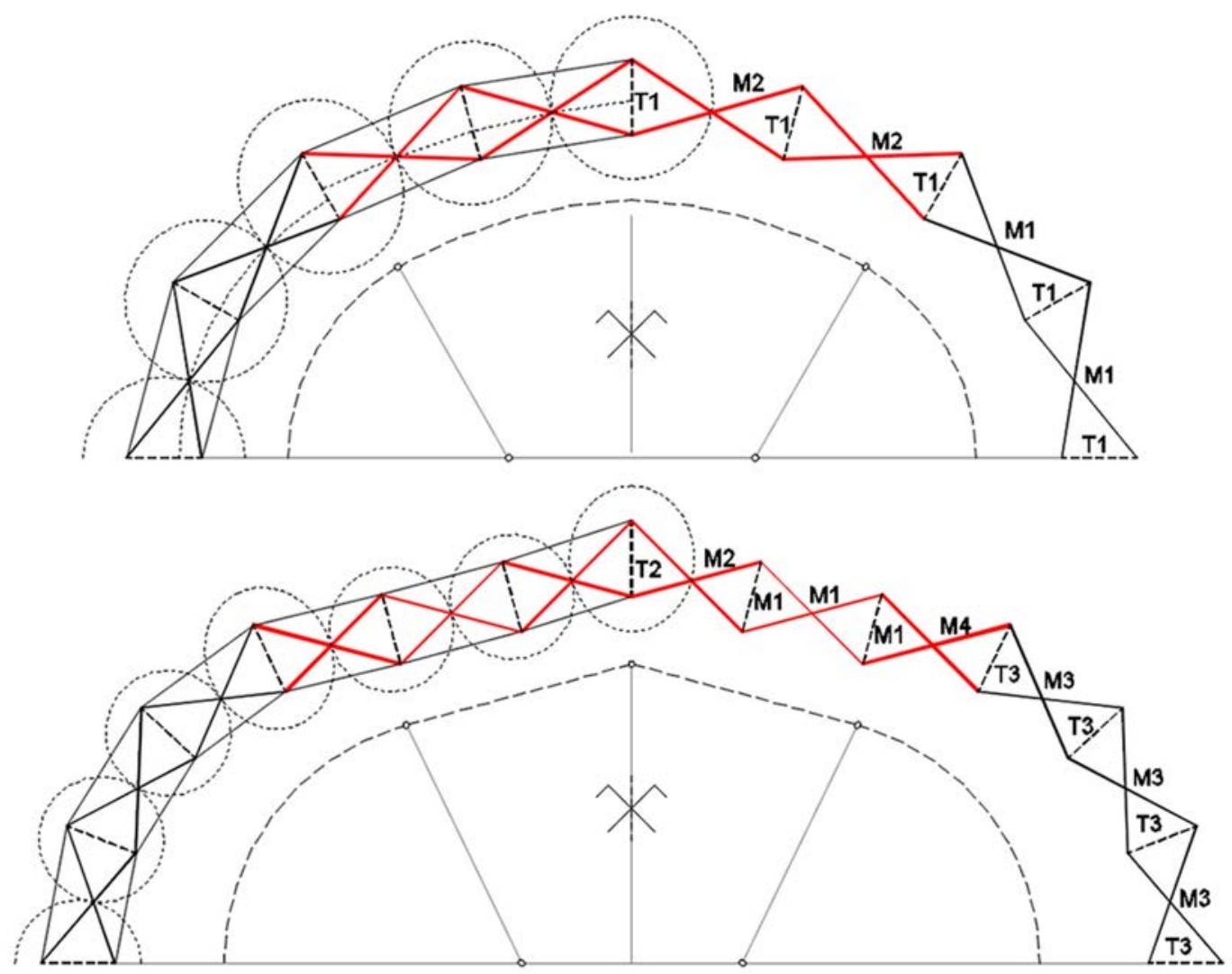

Figura 6. Desplegables de directriz carpanel (arriba) y tudor (abajo).

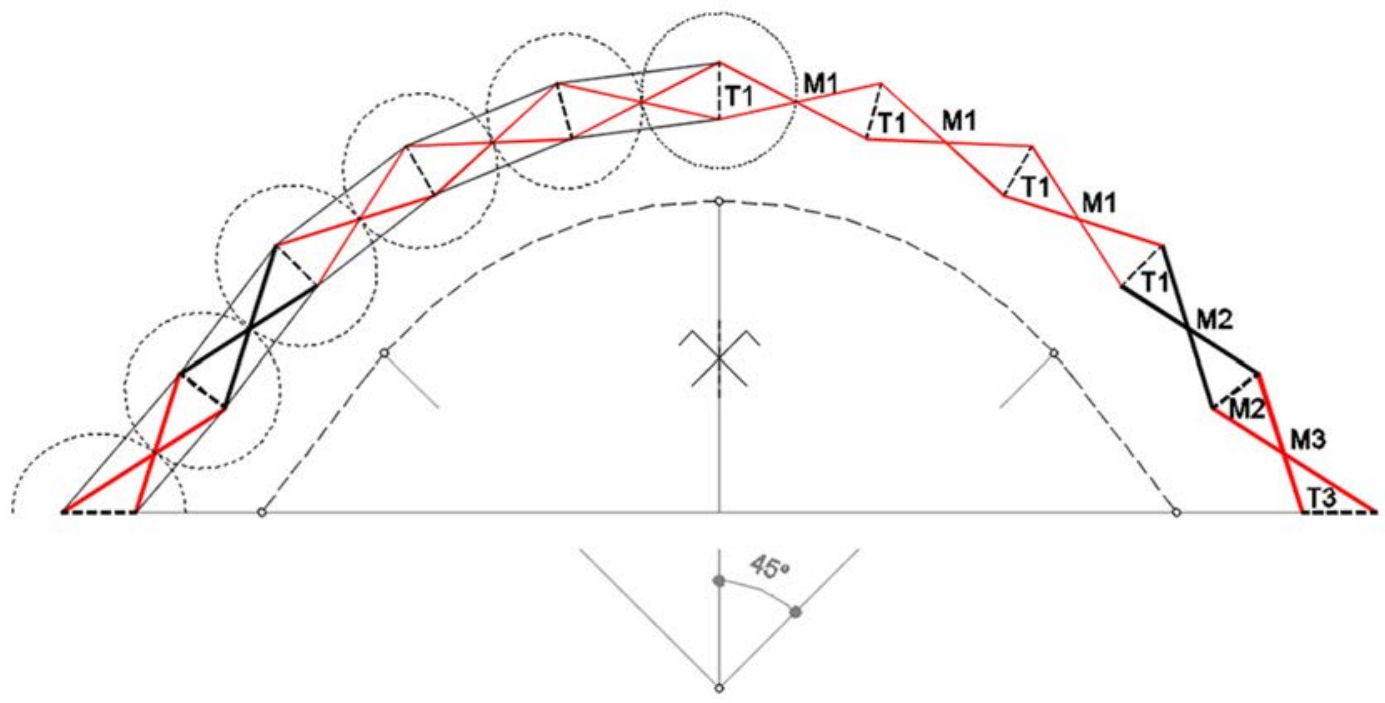

Figura 7. Desplegable de directriz curva con arranques rectos. 
Para la construcción de estas geometrías existen dos vías: optar por mantener la continuidad de la curva directriz o priorizar la continuidad de las curvas de trasdós -intradós y extradós-. La primera de ellas implica que todos los nodos se encuentren sobre la directriz escogida pero da lugar a cantos ligeramente diferentes entre tramos; en la segunda solución son las articulaciones de enlace entre aspas las que se mantienen sobre las curvas prefijadas, manteniéndose por tanto el canto de la solución a costa de un ligero salto en la directriz. Con este último criterio se ha realizado la desplegable carpanel, mientras la desplegable tudor -combinación de curvas y rectas- se ha realizado con aquél: si bien se puede resolver la transición curva-recta manteniendo las curvas de trasdós, la inclusión del vértice obliga a aplicar el criterio de continuidad de la directriz.

En la carpanel, escogido este trazado, el punto de tangencia $-60^{\circ}$ en la Figura 6, arriba- y el número de módulos de cada tramo -2 en cada arranque y 4 en el centro en la Figura 6, arriba-, fijados los radios interior y exterior y una vez trazados los módulos del arranque circular, el problema pasa por posicionar el punto de corte de aspas del módulo adyacente -nodo, punto de articulación tipo tijera- y el radio de una de las superficies de trasdós. La exigencia de igualdad longitudes (l1 + l2 = l'1 + l'2), equivale a obligar a que los nodos de la solución se encuentren sobre elipses cuyos focos son los nudos de contacto entre aspas, elipses que aparecen dibujadas en las figuras. Esta propiedad es la que permite el trazado gráfico de la desplegable.

Para mejorar las condiciones de evacuación de agua -el punto más alto tiene tangente horizontal-se la dotó de pendiente en la clave introduciendo un vértice construido prolongando las barras que concurren en los nudos superiores de las aspas que se obtienen al dividir el arco central en un número impar de módulos -siete en la Figura 8-, definiendo un sistema de aspas múltiples. Los modelos realizados confirmaron que la solución, si bien funciona adecuadamente desde el punto de vista cinemático, se complica porque el número de barras aumenta apreciablemente, dependiendo además la pendiente obtenida en la clave de la división de módulos realizada.

En el caso de la directriz formada por combinación de curvas y rectas, se pueden seleccionar la pendiente de la parte inclinada (o el ángulo cubierto por la parte curva, es lo mismo), el número de módulos de la parte recta y de la parte curva, así como la apertura de uno de los tipos de módulos (el otro es derivado).

La solución ha mostrado un adecuado funcionamiento cinemático -plegado y desplegado satisfactorio-, como pone de manifiesto la Figura 9, en el que cada tramo está resuelto con tres módulos, con una apertura de $30^{\circ}$ los del tramo recto, cubriendo los arranques $74,9^{\circ}$ y disponiéndose los tramos rectos con una inclinación de $15,10^{\circ}$.

En el caso de emplear un único módulo específico para la parte recta se obtiene una aproximación cinemáticamente viable al perfil del arco apuntado (Figura 10), que sin esta modificación es una solución incompatible.

La combinación de las distintas variantes enumeradas apuntadas anteriormente -resolución del vértice, directrices formadas por varios tramos rectos y curvos, incluso con distintos tramos de curvatura- permiten formular sorprendentes propuestas de desplegables cinemáticamente admi-

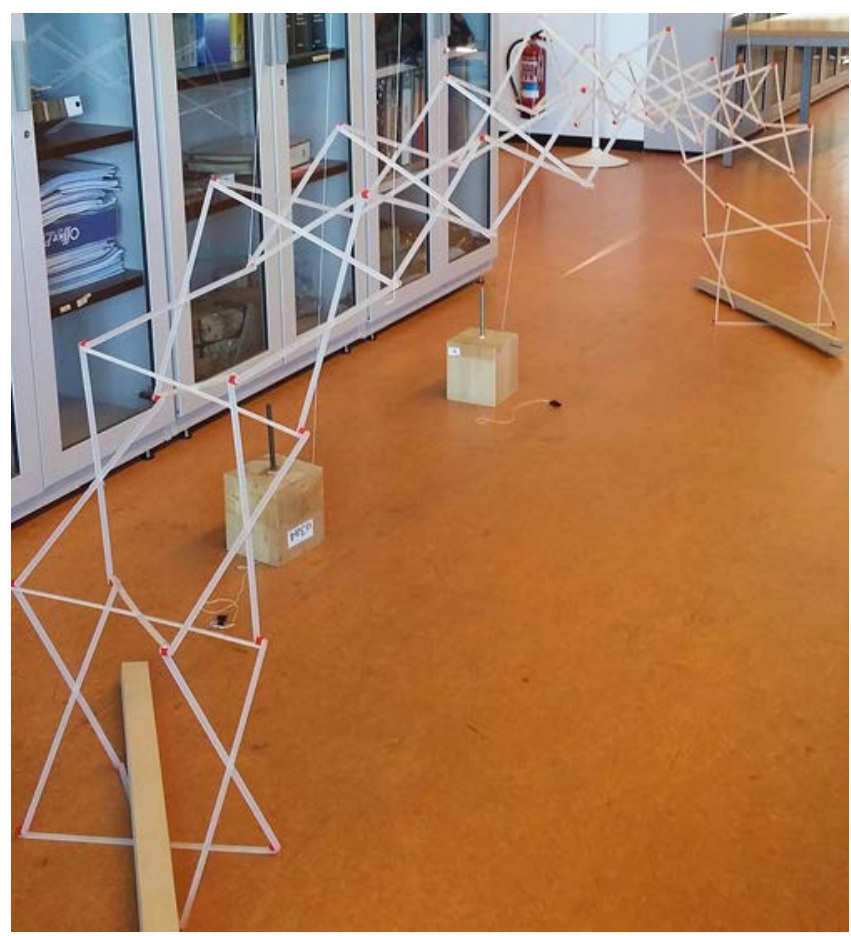

Figura 9. Modelo de desplegable tudor.

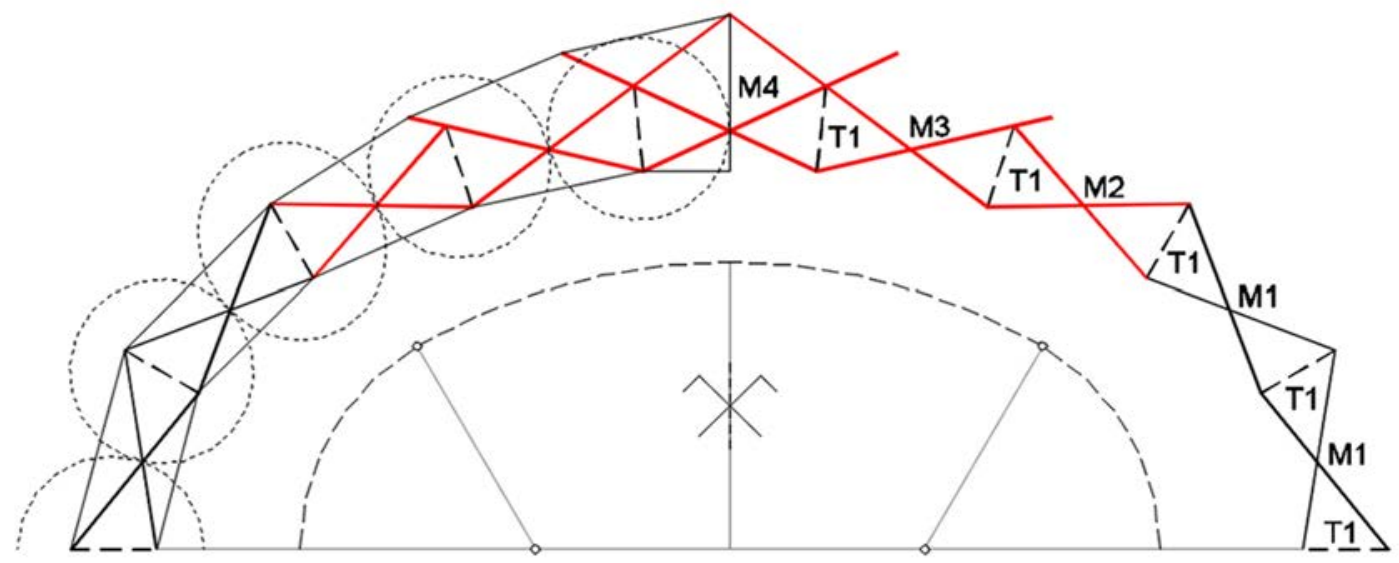

Figura 8. Desplegable carpanel con extradós apuntado. 


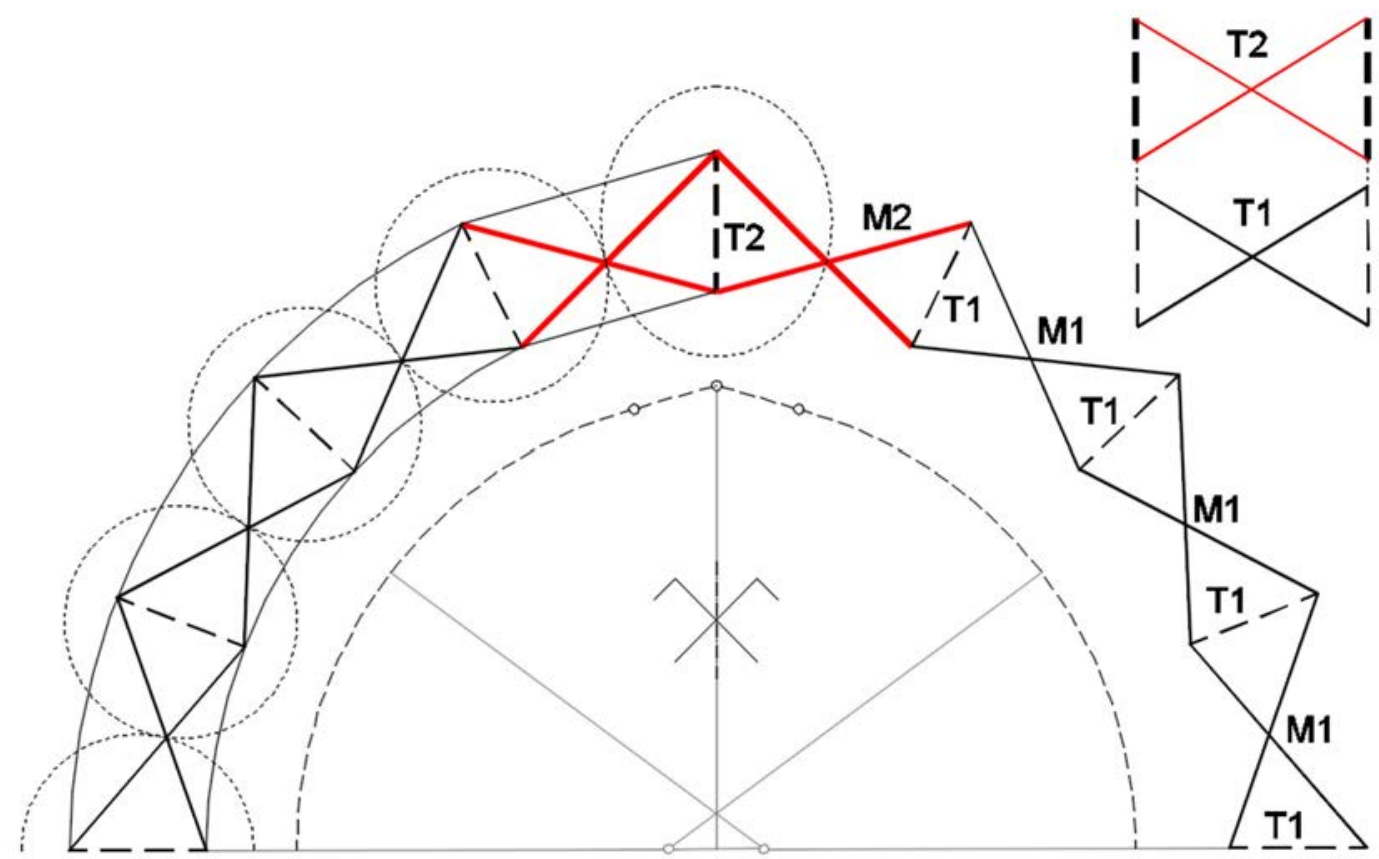

Figura 10. Desplegable de directriz apuntada.

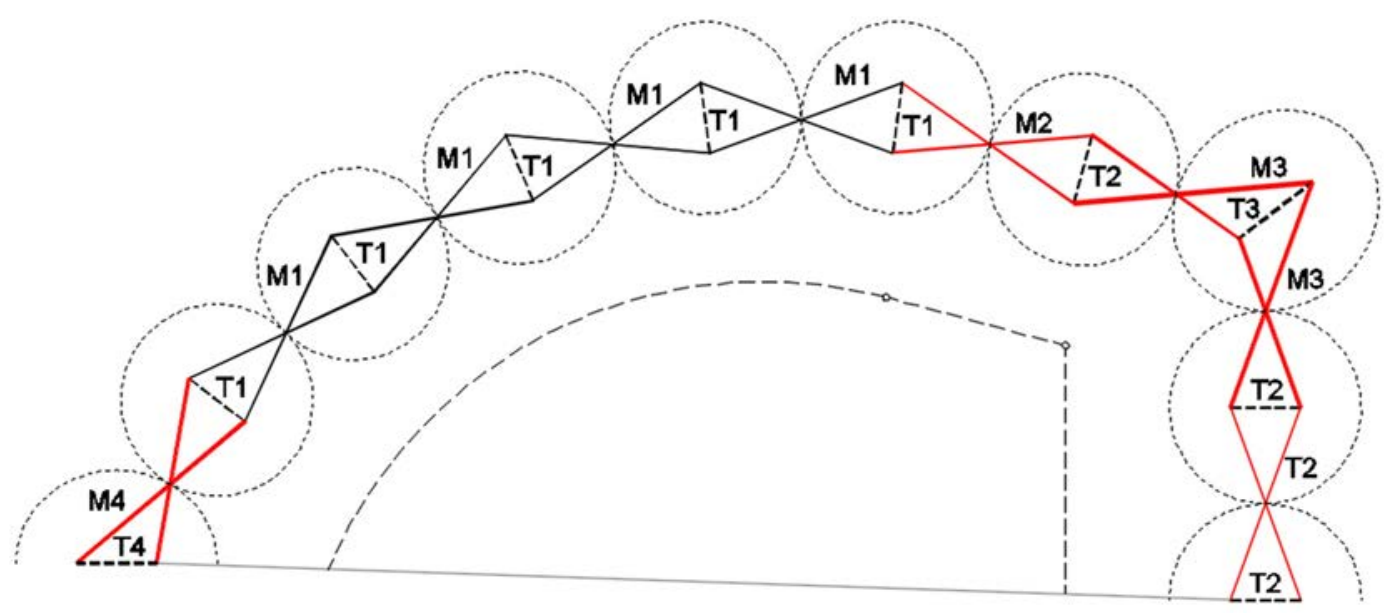

Figura 11 Desplegable de directriz combinada.

sibles (Figura 11), ampliando notablemente las soluciones posibles.

\section{MEJORAS MECÁNICAS}

Se entiende por acodalamiento la situación en la que sendas barras de dos aspas contiguas se encuentran alineadas, formando parte de la misma recta. Esta situación se puede producir en mallas planas en las que existan uno o varios vértices o en mallas cilíndricas cuando el semiángulo de apertura de las barras del aspa iguala el ángulo que en sección cubre el semimódulo (10).

Los modelos cinemáticos han puesto de manifiesto que la situación de acodalamiento es una situación límite que corresponde a la luz máxima cubierta por la desplegable: hasta alcanzarla la estructura está en fase de apertura -incremento de luz-, superado este límite la estructura cambia de forma, reduce su luz y aumenta su flecha.

Si, en una situación de acodalamiento, a la articulación de concurrencia se añade un mecanismo de bloqueo, el conjun- to resultante mejora notablemente su capacidad portante a compresión -véase en (2) el empleo de las situaciones de acodalamiento como forma de mejorar la capacidad portante de la solución -Ejemplos T, U y, especialmente, V (2) pp. 14, 16, 50-54 y 63-94-.

Las consideraciones anteriores han llevado a realizar una serie de diseños de desplegables basándose en esta situación. Así se ha desarrollado el modelo de la Figura 12, que conceptualmente puede obtenerse a partir de sendos faldones planos (de $22,5^{\circ}$ de inclinación) formados cada uno por dos módulos sobre sendos arranques circulares (de $45^{\circ}$ ) resueltos cada uno con un módulo o bien concibiendo la estructura como un conjunto de cuatro planos simétricos resueltos con dos módulos cada uno de los centrales, mientras los extremos se resuelven con un módulo cada uno.

Ello da como resultado una estructura en la que se producen tres situaciones de acodalamiento, dos inclinadas en los arranques y una horizontal en la clave. El modelo a escala pone de manifiesto que si bien en los arranques se alcanza 
sin problemas el acodalamiento, no se llega a alcanzar éste en la posición horizontal superior como consecuencia de la deformación de la estructura, por lo que en la realidad habría que empujar estos nudos hasta su posición teórica como se hizo por ejemplo en la piscina de San Pablo en Sevilla (9) (12)

Es difícil diferenciar visualmente estas estructuras: si se comparan las imágenes de las Figuras 12 y 15, la primera impresión es que se trata de la misma estructura: sólo fijándose en los detalles -forma de las aspas, $\mathrm{n}^{\mathrm{0}}$ de ellas transversales- se perciben las diferencias. Esta misma confusión puede producirse entre el modelo anterior y otros modelos conocidos basados en la directriz circular. En la Figura 13 adjunta se di- bujan superpuestas ambas soluciones, tudor y circular, para una misma luz libre, lo que aclara las diferencias.

La comparación de los trazados evidencia que el ángulo de la cobertura en el vértice es de $15^{\circ}$ en la solución basada en la circunferencia frente a los $24,55^{\circ}$ con los que se ha diseñado esta solución y que la disminución de altura de la estructura es del 26,35\%, si bien presenta una mayor apertura de aspas para la situación de acodalamiento.

Que las estructuras desplegables sean mecanismos es una condición necesaria para que se pueda producir el despliegue, pero en el caso de las desplegables cilíndricas de base cuadran-

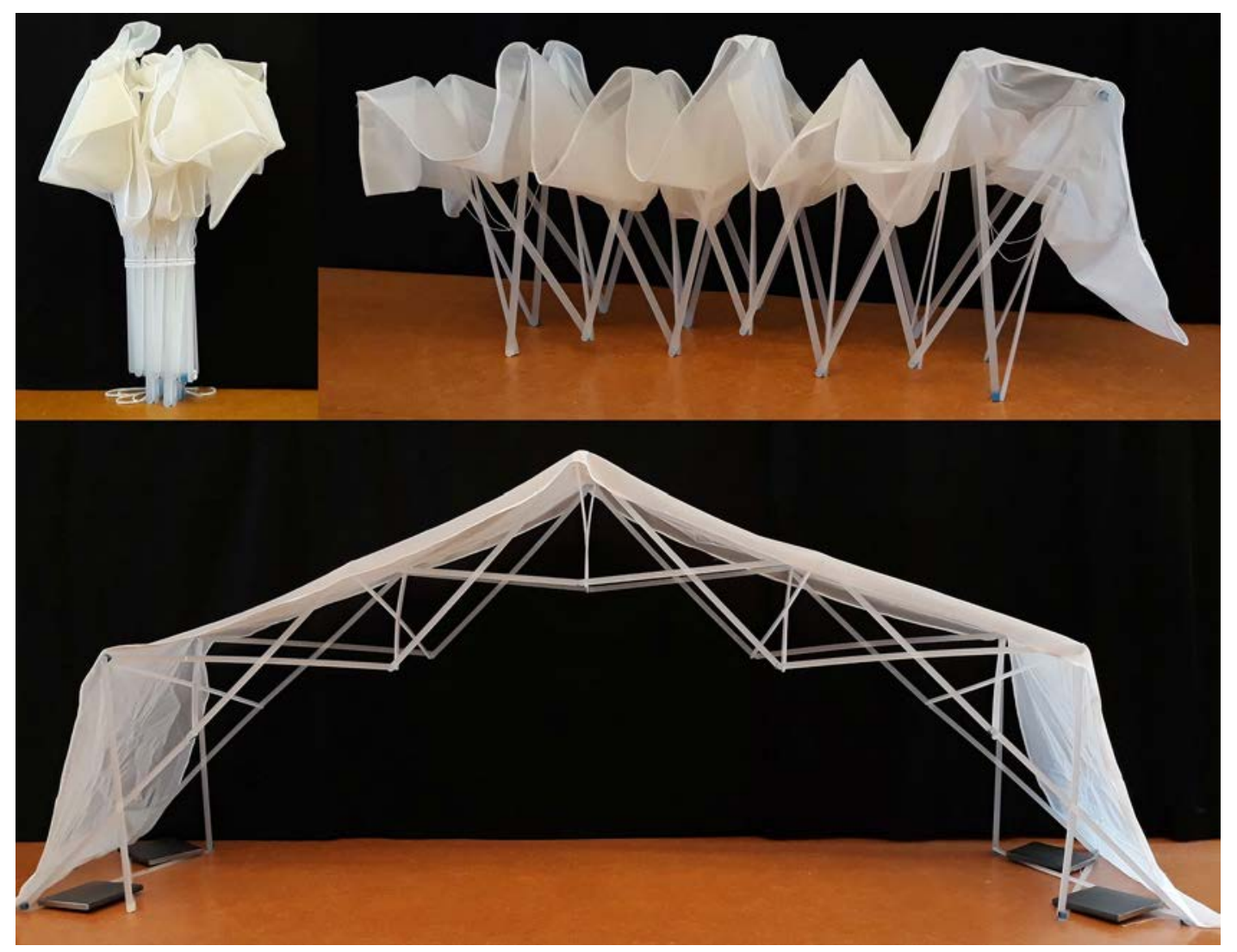

Figura 12. Desplegable de directriz tudor.

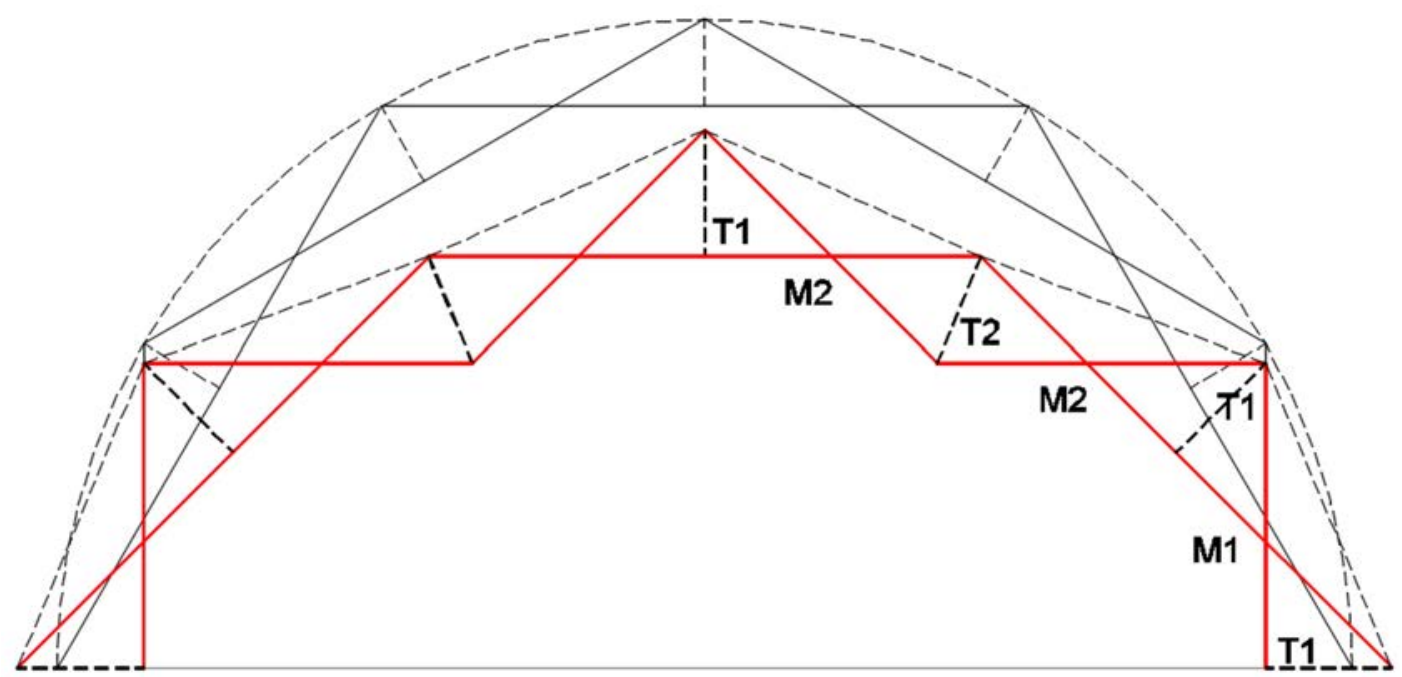

Figura 13. Comparación de trazados de la directriz tudor de la Figura 12-y la semicircular. 
gular el mecanismo se produce también en sentido transversal en planta: las articulaciones de los nudos son rótulas transversales con una capacidad de rotación limitada -dependiente de la holgura del nudo- por lo que la estructura carece de la necesaria rigidez transversal y tiende a adoptar configuraciones en planta con forma de S. Esta situación limita apreciablemente el número de barras de las aspas que forman la directriz de la estructura, dado que la deformabilidad transversal de ésta aumenta con el número de nudos empleados.

Una forma de afrontar este problema es conferir a la cobertura una función arriostrante enlazándola con los nudos extremos de las aspas antes del despliegue (12). Sin embargo, resulta problemática la construcción de los arranques. Además, exigen al nudo la existencia de una superficie superior para el anclaje de la tela que no quede por debajo de los bordes de las barras que concurren en el nudo.

Otra posibilidad es colocar sendos cables según las diagonales (superiores) de cada recuadro -formando una cruz de San Andrés cuya longitud ayuda a fijar el despliegue en el punto deseado, y que tiene la ventaja de facilitar la sustitución de la cobertura en caso de rasgado. Finalmente, en los modelos a escala (Figura 12) se ha empleado una solución mixta, mediante cables en los recuadros extremos -los más determinantes a la hora de mantener la estructura en su sitio- y con el textil de cubierta en el resto. Esta solución facilita la realización de los bordes, al tiempo que libera a la parte inferior de la tela del requisito estructural, permitiendo soluciones de encuentro con el terreno mucho más flexibles. Sin embargo, en el modelo anterior, la cobertura tiende a separarse del nudo intermedio del faldón -tira de éste hacia arriba- dado que queda ligeramente más bajo que la línea que une los dos nudos extremos. El trabajo con modelos de mayor detalle permitirá conocer con precisión este y otros efectos.

\section{EXPLORANDO LAS SUPERFICIES ENVOLVENTES}

Los módulos de aspas asimétricos posibilitan sugerentes juegos formales en las desplegables, pero también permiten dar respuesta a nuevos problemas, en este caso resolver una desplegable con curvas de trasdós y extradós diferentes: una envolvente exterior a base de faldones planos para facilitar la evacuación de aguas, el arriostramiento y la colocación de la cubierta y una superficie interior cilíndrica de área mínima, esto es una cilíndrica recta de base semicircular. Se generan así soluciones de espesor variable.

Se han dibujado propuestas que emplean dos y tres módulos por cada faldón plano (Figura 14). El diseño parte de la situación de acodalamiento de barras, con arriostramientos transversales construidos con un solo aspa en las zonas de mayor distancia entre articulaciones y con dos en el caso de las zonas de articulaciones próximas. Las propuestas estudian el espacio entre trasdoses que resulta en cada caso.

En las estructuras de dos módulos por cada faldón plano con arranques de uno -división del cuadrante en tres sectores, cubriendo cada módulo $30^{\circ}$, Figura 14.a-, la geometría de la desplegable se obtiene de forma gráfica directamente; sin embargo en el caso de tres módulos por faldón, Figura 14.c, es preciso un cálculo iterativo que defina los módulos para cumplir las condiciones de plegabilidad. Siempre es obligado el cálculo de las longitudes de los módulos transversales para permitir la apertura y cierre.
En la desplegable de 6 módulos - uno por cada arranque y dos por cada faldón inclinado, Figuras 14.a y 15-, las comprobaciones geométricas arrojan una pequeña incompatiblidad con la premisa de obtener una superficie plana: el nudo de concurrencia de las dos aspas de arriostramiento transversal sobresale muy ligeramente del plano del faldón, con un desajuste del 0,5\% sobre el intereje entre pórticos; sin embargo la realidad experimental muestra que la situación es justo la inversa y no llega a alcanzar la cota que correspondería al plano del faldón; en el caso de la de 8 módulos -faldones que cubren $45^{\circ}$, resueltos con 2 módulos por faldón, Figura 14.b-, el desvío sobre la coplanariedad baja al o,01\%. En todo caso el comportamiento cinemático ha sido plenamente satisfactorio.

En el caso de las de tres módulos por faldón (Figura 14.c), las condiciones de plegabilidad conducen a que los módulos del arriostramiento transversal integrado por dos módulos sean además asimétricos, hecho que conduce a que los nudos transversales se salgan del plano un 3,11\% de la separación entre los planos de desplegables directrices.

\section{DESPLEGABLES ROMBOIDALES: DESPLEGABLES CON DESPLIEGUE AL BIES}

Las necesidades de arriostramiento expuestas con anterioridad se encuentran en el origen de estas propuestas: se trató de convertir las aspas de arriostramiento -elementos soportados con misión estabilizadora- en elementos portantes girando ambos sistemas de aspas con respecto al eje del recinto, con lo cual los dos sistemas de aspas definen rombos en planta -cuadrados en el caso de encuentros perpendiculares-. La concordancia de cotas de la solución viene garantizada por las condiciones de simetría aplicadas al trazado, esto es, esta solución tiene la ventaja que elimina las condiciones transversales de plegabilidad, por lo que las condiciones de plegado se convierten en un problema plano.

La condición de compatibilidad geométrica en el despliegue obliga a que los nudos estén contenidos en el plano de la estructura, lo cual lleva a que los nudos comunes de los rombos estén situados en una misma vertical. Se obtienen así desplegables de tipo traslacional (11) en las que con un mismo módulo se puede construir toda la estructura si se admite que el apoyo en el borde se produzca en un solo y que las aspas sean asimétricas.

Se ha construido un modelo cinemático formado por sendos sistemas de pórticos desplegables a dos aguas colocados en planta con una inclinación de $\pm 45^{\circ}$ con respecto al eje de la nave, generando así una cubierta a dos guas. Para los ángulos anteriores, los encuentros entre pórticos se producen de forma perpendicular, por lo que se puede emplear el nudo desarrollado. Con este tipo de nudo el empleo de otras inclinaciones complica el diseño e incrementa su tamaño para evitar las interferencias durante el despliegue. Así para dos familias de aspas que formen un ángulo en planta de $60^{\circ}$ entre sí es necesario un nudo hexagonal que aumenta en un $70 \%$ la separación entre barras de la misma familia de aspas -aquellas que en el dibujo conceptual estarían contenidas en el mismo plano-.

Todo el conjunto se construye con únicamente dos barras, tal y como se aprecia en la Figura 16. El diseño fue realizado para la teórica posición de acodalamiento horizontal, por la facilidad de control visual del despliegue que introduce y para posibilitar la implementación de mecanismos de bloqueo de 


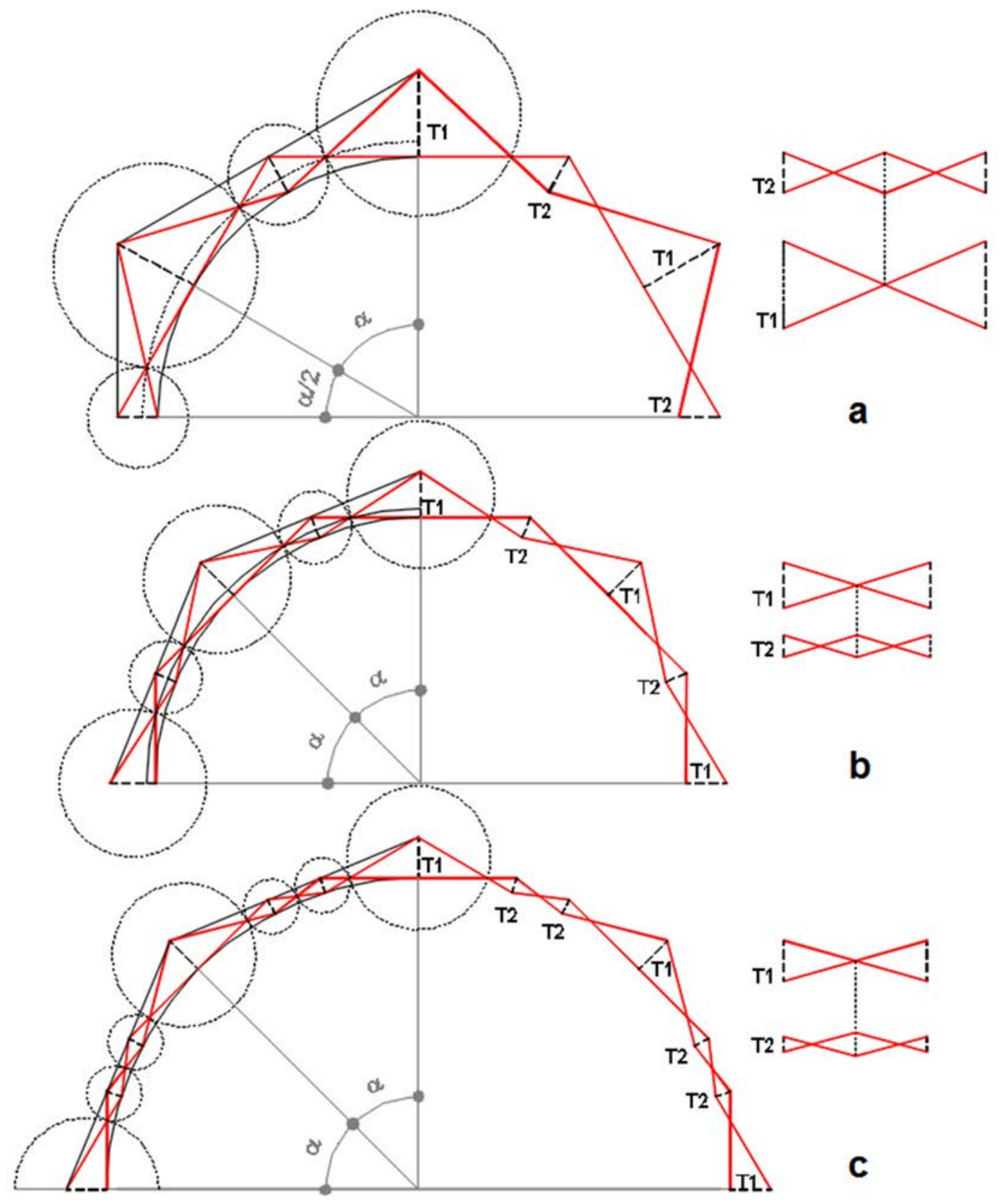

Figura 14. Desplegables con intradós circular y extradoses planos.

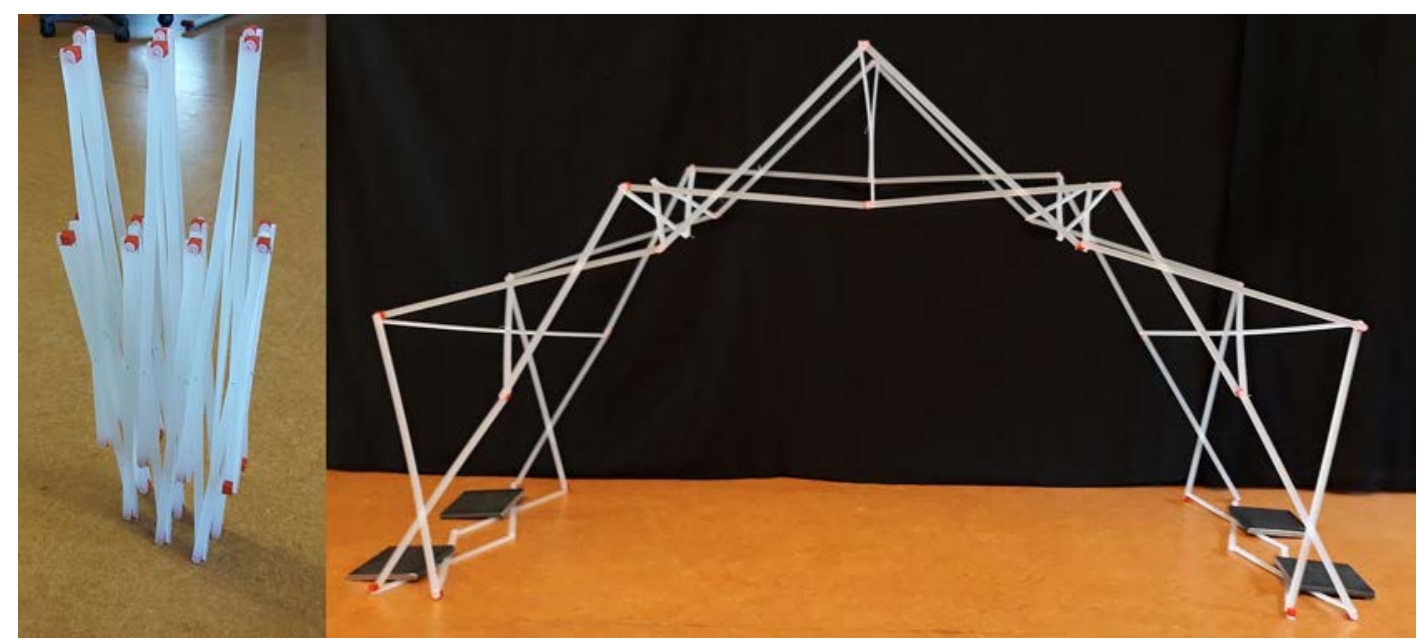

Figura 15. Desplegable con intradós circular y extradós de faldones planos de 6 módulos de la Fig. 14.a. 
nudos. En la situación plegada el paquete de barras tiene forma escalonada, apoyando sobre el suelo exclusivamente los nudos perimetrales.

La solución tiene una rigidez -longitudinal y transversalmuy notable, manteniendo su forma durante todo el despliegue y estabilizándose en cualquier fase hasta casi el final del despliegue: sólo para mantener la apertura máxima hace falta disponer tirantes o añadir barras de rigidización en los bordes. Por el contrario, los testeros de la solución a dos aguas quedan definidos por una serie de nudos, pero no de barras, lo que obligaría a añadir éstas para su remate.

Si se invierte la colocación de las barras de las aspas del testero, se llevan al suelo los nudos del borde de éste, dando lugar a un nuevo faldón y configurando así cubiertas desplegables a 3 ó 4 aguas construidas exclusivamente con dos tipos de barras (Figura 16, en la que se han añadido cuatro barras en las esquinas para fijar la posición de despliegue).

Se puede superar el límite de acodalamiento durante el despliegue a costa de realizar un aporte energético extra. Si bien se reducen el canto y la luz -se incrementa la altura de cumbrera-, la estructura mantiene su compatibilidad todo el tiempo.

La disposición de barras al bies se puede aplicar también a desplegables de directriz cualquier curva para conseguir una bóveda. Sin embargo la aplicación es algo más complicada: por una parte, parece obligada su aplicación a directrices cir-

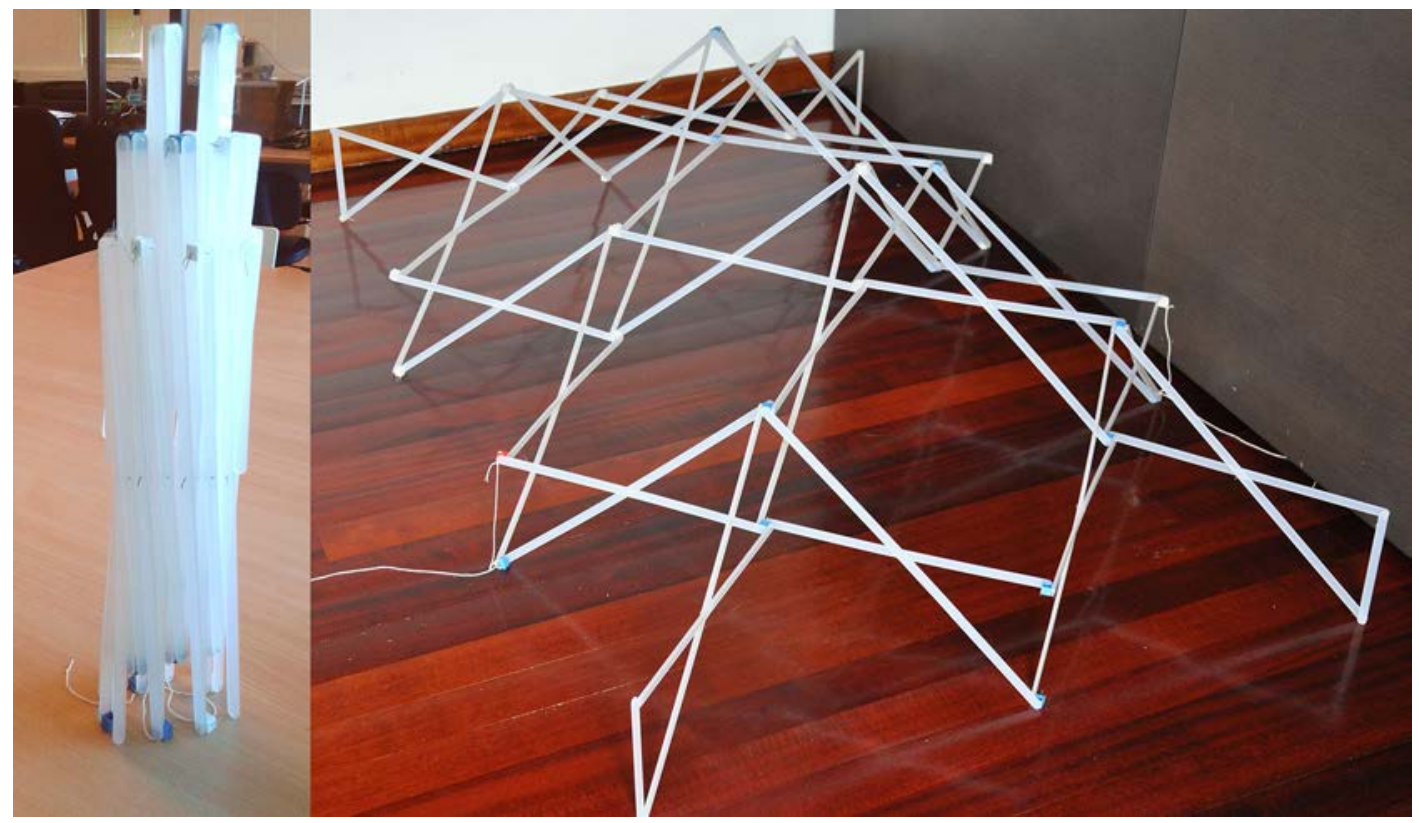

Figura 16. Desplegable de perfil quebrado formada por sistemas de aspas dispuestos a $45^{\circ}$.

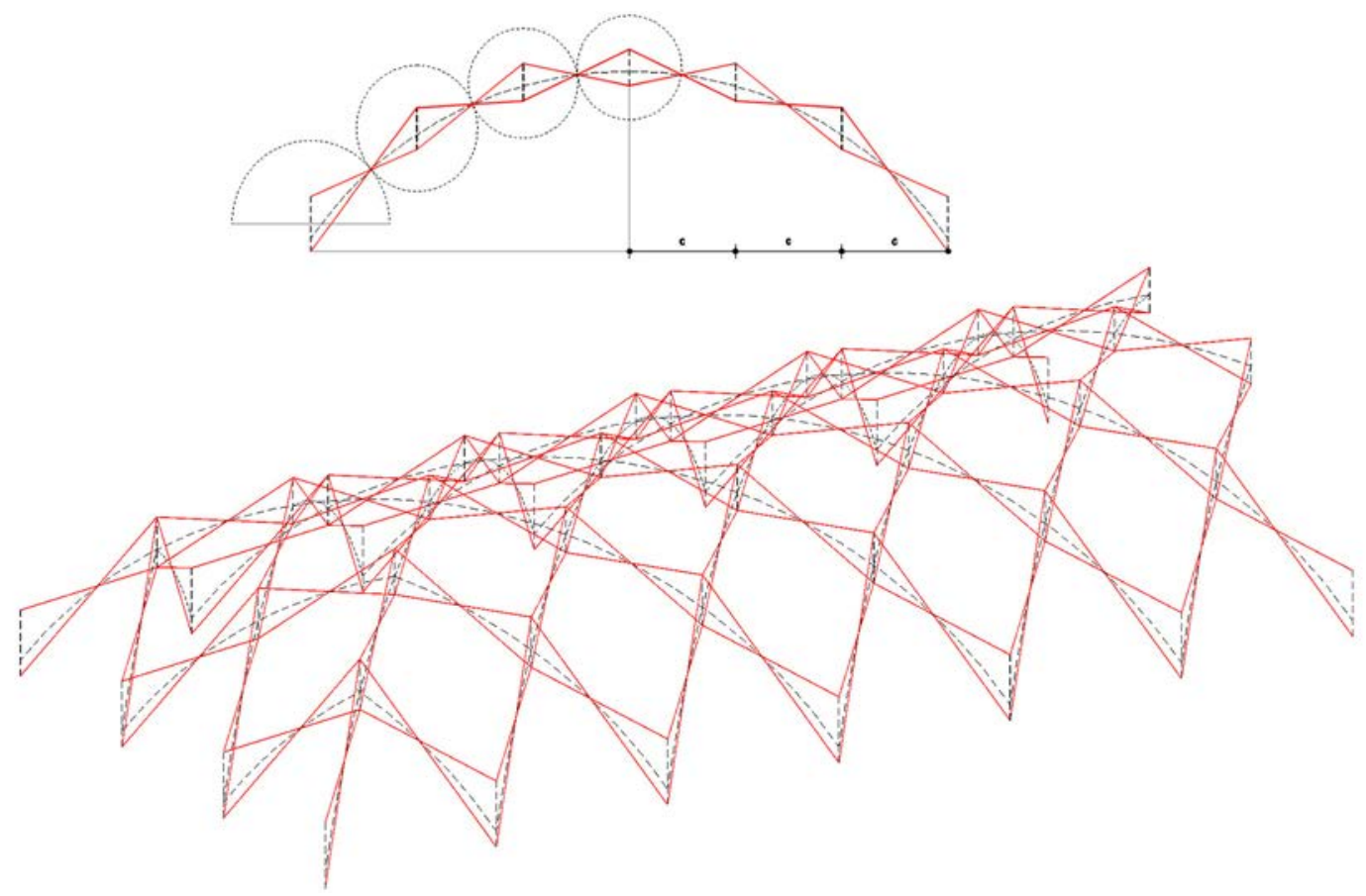

Figura 17. Desplegables de directriz curva formadas por sistemas de aspas dispuestos a $45^{\circ}$. 
culares rebajadas para la formación de recintos -la disposición al bies reduce la luz libre-, y, por otra, hay que fijar la posición de cada elemento vertical atendiendo a las condiciones de plegabilidad, resultando que todas las aspas del semiarco son distintas. Para desplegables con una apertura en torno a $120^{\circ}$, se han estudiado trazados atendiendo a tres criterios diferentes, pero manteniendo siempre constante la directriz rebajada. Los criterios son:

a) distancia horizontal entre las verticales de conexión de aspas constante,

b) disposición de los nodos sobre la directriz y los nudos sobre la superficie exterior

c) longitud máxima de las barras constante.

De estos tres criterios, sólo es posible la aplicación sistemática del primero en desplegables al bies. La Figura 17 muestra un ejemplo de su aplicación.

\section{CONCLUSIONES}

- Las estructuras desplegables son una solución sugerente para habilitar refugios de emergencia. Su empleo en cualquier climatología pasa por desarrollar cubiertas con buenas condiciones de evacuación de aguas, poniendo el foco en la las soluciones de cubiertas desplegables inclinadas. Para ello es necesario afrontar la introducción de un quiebro en la directriz de las desplegables de aspas de base cuadrangular.
- La capacidad para resolver quiebros ha permitido proponer un conjunto de nuevas propuestas para este tipo de desplegables despegables entre las que se encuentran las desplegables de directriz poligonal, las de perfil oval, las formadas por arcos y tramos rectos con o sin vértice intermedio, las configuraciones en arco apuntado o nuevas soluciones para desplegables cilíndricas rebajadas, demostrándose su viabilidad geométrica y cinemática través de los modelos construidos. En éstos se incorporan también distintas soluciones de arriostramiento y se estudian sus efectos de forma cualitativa.

- Se identifica una nueva clase de desplegables polares cuadrangulares con distintas envolventes de intradós y extradós -desplegables de espesor variable-, incrementando las posibilidades arquitectónicas de estas estructuras. Se demuestra también su viabilidad geométrica y cinemática.

- Como forma de eliminar la dicotomía entre sistema de aspas de arriostramiento y sistema de aspas portantes, se formulan propuestas formadas por un único sistema de aspas girado en planta (desplegables al bies). Si bien las condiciones geométricas de plegabilidad obligan a que las aspas sean de tipo traslacional, esta disposición elimina las restricciones de plegabilidad transversal, por lo que las únicas limitaciones de plegabilidad se restringen al plano. Esta situación permite resolver cubiertas a 2, 3 y 4 aguas de espesor constante, y cilíndricas de cualquier directriz.

\section{REFERENCIAS}

(1) Escrig, F., Valcárcel, J. P. (1988). Estructuras espaciales desplegables curvas. Informes de la Construcción, 39(393): 53-71. https://doi.org/10.3989/ic.1988.v39.i393.1606

(2) Hernández Merchan, C.H. (1987). Deployable Structures (Unpublished Master Thesis). Master Thesis of Science in Architecture Studies. Boston: Massachusetts Institute of Technology. MIT Libraries Document Services.

(3) Escrig, F. (1985). Expandable space structures. Space Structures Journal, 1(2): 79-91. https://doi.org/ 10.1177/026635118500100203

(4) Escrig, F., Valcarcel, J.P. (1987). Curved expandable space grids. En Proceedings of the international Conference on the Design and Construction of Non-Conventional Structures (vol. 2, pp.157-168) London. https://doi.org/10.4203/ccp.7.18.1

(5) Derya Bakbak, Mustafa Özakça y Mehmet Tolga Göğüş (2016). Development of design methodologies for deployable fabric structures in civil engineering. Journal of the Faculty of Engineering and Architecture of Gazi University 31(1): 73-86

(6) Valcárcel, J.P., Escrig, F. (1994). Pioneering in Expandable Structures: The 'Madrid I' Notebook by Leonardo da Vinci. Bulletin of the international Association for Shell and Spatial Structures, IASS, 35 (114): 33-45.

(7) Valturium, R. (1448). De Re Militari, p. 404 Paris. Libro X, p. 259 (Google Docs). Referido por Jufre Garcia, X. (2017, 3-7 octubre) Los Artificios de Juanelo. La principal infraestructura hidráulica del Renacimiento europeo. En Actas del X Congreso Nacional y II Internacional Hispanoamericano de Historia de la Construcción (pp. 837-847) San Sebastián - Donostia. Universidad del País Vasco (UPV-EHU). Recuperado de http://www.sedhc.es/biblioteca/actas/Jufre\%20 Garcia.pdf

(8) Pérez Piñero, E. (1971). Teatros desmontables. Informes de la Construcción, 24(231): 34-42. https://doi.org/10.3989/ ic.1971.v24.i231.3360

(9) Escrig, F., Valcárcel, J.P., Sánchez, J. (1995). Deployable Structures Squared in Plan: Design and Construction. En Spatial Structrures: Heritage, Present and Future. IASS International Symposium (pp. 483-492). Milano, Italia.

(10) Blanco Marín, Fernando (director). Arquitectura Efímera. Recuperado de https://docs.google.com/presentation/d/1d8 6cKMNBO1WywLIhVObK2zGNYq5Q5h44xxpqoawCmVo/edit\#slide=id.g173176aedb_1_28

(11) Sánchez Cuenca, L. (1996). Geometric models for expandable structures. En Mobile and Rapidly Assembling Structures II (pp. 93-102). Escrig \& Brebbia. Computational Mechanics Publications, Southampton.

(12) Escrig, F., Valcárcel, J., Sánchez, J. (1996). Las cubiertas desplegables de malla cuadrangular. Boletín Académico de la ETSA de La Coruña, (20): 37-46. Recuperado de http://hdl.handle.net/2183/5274 